\title{
Neumonía e insuficiencia renal aguda como complicaciones de la varicela en el adulto
}

\author{
R. BERNABEU MORA, E. RUBIO GIL*, J. NAVARRO FUENTES*, A. CANO \\ SÁNCHEZ*, F. SÁNCHEZ GASCÓN \\ Sección de Neumología. *Servicio de Medicina Interna. Unidad de Enfermedades \\ Infecciosas. Hospital General Universitario. Murcia
}

\author{
PNEUMONIA AND ACUTE RENAL FAILURE COMPLICATING VARI - \\ CELLA IN THE ADULT
}

\section{RESUMEN}

La varicela es una enfermedad propia de la infancia que puede afectar a adultos con serias complicaciones. La neumonía es la más frecuente y grave. La afectación renal está apenas descrita en la literatura. La presentación conjunta de ambas complicaciones es inusual. Presentamos un caso de varicela en el adulto que desarrolló simultáneamente neumonía con distrés respiratorio e insuficiencia renal aguda que evolucionaron favorablemente.

PALABRAS CLAVE: Varicela. Neumonía. Insuficiencia renal aguda.

\begin{abstract}
Varicella is a disease in childhood. When it affects adults, serious complications can develop, the most frequent and most dangerous being pneumonia. Acute renal failure is an exceptional complication which infrequently is reported in relevant current literature. The association of pneumonia with acute renal failure in a patient with varicella is unusual. We report a varicella case in an adult patient who suffered from pneu monia with respiratory distress syndrome and acute renal failure, both of them had a favourable clinical course.
\end{abstract}

KEY WORDS: Varicella. Pneumonia. Acute renal failure.

Bernabeu Mora R, Rubio Gil E, Navarro Fuentes J, Cano Sánchez A, Sánchez Gascón F. Neumonía e insuficiencia renal aguda como complicaciones de la varicela en el adulto. An Med Interna (Madrid) 2002; 19: 136-138.

\section{INTRODUCCIÓN}

La varicela es una enfermedad exantemática producida por el virus varicela-zóster (VVZ), que afecta sobre todo a niños. Puede afectar al 1-2\% de los adultos, siendo entonces las complicaciones y mortalidad 25 veces mayor que en la infancia (1). La neumonía es la más frecuente y grave, presentándose entre el 16-50\% de los adultos infectados con una mortalidad del 10-30\% en inmunocompetentes aumentando hasta el $35-45 \%$ en inmunocomprometidos y embarazadas (2). En pacientes VIH la infección por el VVZ es frecuente, sin embargo la neumonía varicelosa (NV) se ha descrito sólo en casos aislados $(3,4)$. Otras complicaciones son la encefalitis, meningitis aséptica, mielitis transversa, síndrome de Guillain-Barré, artritis, neuritis óptica, necrosis retiniana, uveítis, queratoconjuntivitis, miopericarditis, hepatitis, pancreatitis, apendicitis, glomerulonefritis, orquitis, Púrpura de SchöleinHenoch y trombocitopenia (1). Se presenta un paciente con varicela que desarrolló simultáneamente neumonía con distrés respiratorio e insuficiencia renal aguda que se resolvieron satisfactoriamente.

\section{CASO APORTADO}

Varón de 30 años fumador de 40 cig./día. Consultó por fiebre y lesiones cutáneas generalizadas en distinto estadío evolutivo. Su hija fue diagnosticada 2 semanas antes de varicela. La analítica y radiografía de tórax fueron informadas como normales. Se pautó antitérmicos-analgésicos. A los 2 días, consulta de nuevo por tos seca y disnea, apreciándose en el examen físico posterior taquipnea en reposo a $30 \mathrm{rpm}$ y crepitantes en bases pulmonares. Un nuevo estudio radiográfico de tórax objetivaba un infiltrado intersticio-alveolar en campos medios e inferiores (Fig. 1). En el hemograma: leucocitos: $5800 / \mathrm{mm}^{3}$ (S-65, L-22, M-11) y plaquetas: $89000 / \mathrm{mm}^{3}$. Resto de analítica normal. La gasometría arterial (aire ambiente): $\mathrm{pH}: 7,46$. $\mathrm{PO}_{2}: 61 \mathrm{~mm} / \mathrm{Hg}$. PCO2: $34 \mathrm{~mm} / \mathrm{Hg}$. Sat. $\mathrm{O}_{2}: 92 \%$. El paciente fue trasladado a la UCI, iniciándose tratamiento con Aciclovir 15 $\mathrm{mg} / \mathrm{kg} / \mathrm{iv} / 8$ horas no precisando respiración asistida. En las primeras 48 horas de su estancia en UCI presentó deterioro respiratorio que se tradujo en la radiografía con infiltrado difuso y derrame pleural bilateral (Fig. 2). La gasometría arterial $\left(\mathrm{FiO}_{2}=0,4\right)$ : pH: 7,35. PO2: 51 $\mathrm{mm} / \mathrm{Hg}$. PCO2: $46 \mathrm{~mm} / \mathrm{Hg}$. Sat. $\mathrm{O}_{2} 83 \%$. La analítica mostró: leucocitos: $10500 / \mathrm{mm}^{3}$ (S-62, L-23, M-13), plaquetas: $83000 / \mathrm{mm}^{3}$, GOT: 62 U/L, GPT: $160 \mathrm{U} / \mathrm{L}, \mathrm{GGT}: 114 \mathrm{U} / \mathrm{L}, \mathrm{LDH}: 777 \mathrm{U} / \mathrm{L}$, creatinina: 2,7 mg/dL, BUN: $30 \mathrm{mg} / \mathrm{dL}$, proteinuria de $150 \mathrm{mg} / \mathrm{dL}$ y microhema-

Trabajo aceptado: 7 de septiembre de 2000

Correspondencia: R. Bernabeu Mora. Sección de Neumología. Hospital General Universitario. C/ Marqués de los Vélez, s/n. 30008 Murcia. 


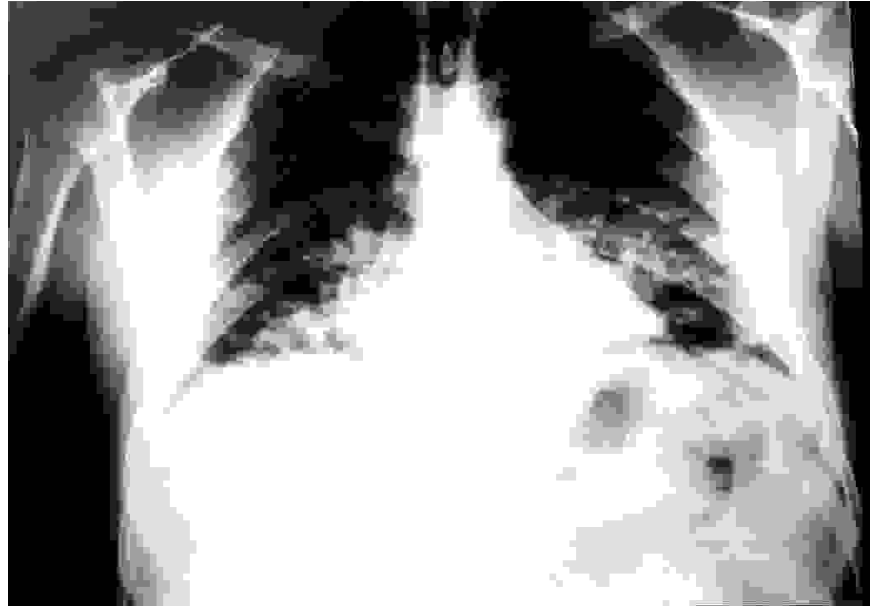

Fig. 1. Radiografía de tórax que muestra un infiltrado intersticioalveolar con componente nodular en bases y región peribiliar practicada en el momento del ingreso.

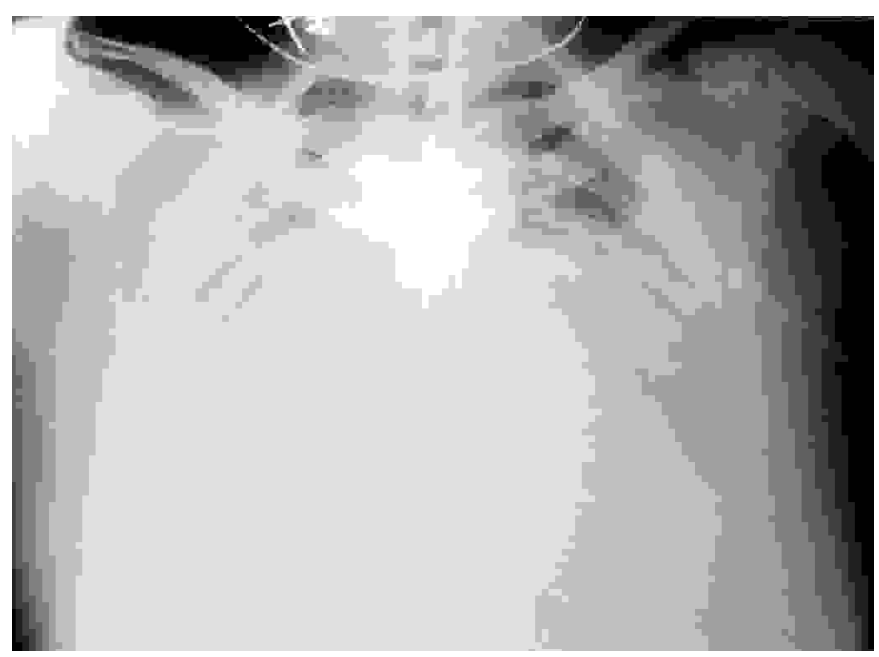

Fig. 2. Empeoramiento radiográfico con infiltrado difuso y derrame pleural bilateral en las primeras 48 horas de su estancia en $\mathrm{UCI}$.

turia de 5-12 hematíes/campo. A partir del $5^{\circ}$ día comienza con mejoría clínica y analítica. En el estudio serológico los anticuerpos para VVZ fueron positivos y para VIH negativos.

\section{DISCUSIÓN}

La NV presenta como principales factores predisponentes el tabaquismo, tercer trimestre de embarazo e inmunosupresión $(5,6)$. En nuestro paciente el único factor de riesgo era el hábito tabáquico. En la mayoría de los casos, se constata el contacto previo con pacientes afectos de varicela como sucedió en el nuestro (7). La sospecha diagnóstica se establece mediante la presencia de un cuadro febril con lesiones cutáneas generalizadas en diferentes estadíos evolutivos. La NV se suele presentar al $3^{\circ}-4^{\circ}$ día del inicio de la erupción cutánea, con tos poco productiva y disnea, siendo menos frecuente el dolor torácico y hemoptisis (2), al igual que ocurrió en el caso descrito. Existe disociación clínico-radiológica, siendo los hallazgos de la exploración física escasos en comparación con el grado de afectación radiológica. El patrón radiológico se caracteriza por un infiltrado intersticial con nódulos que puede progresar hacia la formación de áreas de condensación en bases y zonas perihiliares (8), pudiéndose acompañar de derrame pleural y adenopatías hiliares $(2,1)$. Además, se ha observado neumotórax, enfisema mediastínico y subcutáneo. Tanto el patrón nodular típico como el derrame pleural los presentaba nuestro enfermo. La evolución va desde casos asintomáticos y con curación espontánea hasta otros con SDRA y muerte (9). La hipoxemia grave temprana es el principal factor de mal pronóstico, siendo también importante que el tratamiento se inicie precozmente (10). El Aciclovir iv con dosis de $5-10 \mathrm{mgs} / \mathrm{kg} / 8$ horas es el tratamiento en las formas con sintomatología respiratoria y/o hipoxemia, embarazadas e inmunodeprimidos. Consigue respuestas favorables en 24-48 horas y una resolución completa radiológica antes de la $2^{\mathrm{a}}$ semana (8), se han descrito no obstante, calcificaciones pulmonares años después del proceso con más frecuencia en los fumadores (11). Nuestro paciente, a pesar de iniciar tratamiento antiviral a dosis más elevadas como recomiendan otros autores (12), evolucionó inicialmente hacia insuficiencia respiratoria y distrés respiratorio con mejoría a partir del quinto día. En casos asintomáticos sin hipoxemia y con afectación radiológica, el Aciclovir oral administrado en las primeras 72 horas puede acortar la duración de la enfermedad (13). En fumadores con formas subclínicas es más discutible la indicación de antiviral (14). La duración del tratamiento oscila entre 5-7 días. En la infección por VVZ la aparición de manifestaciones renales es poco frecuente y suelen recogerse casos aislados en la literatura. Las complicaciones renales incluyen glomerulonefritis, fracaso renal agudo secundario a rabdomiólisis y mioglobinuria en el curso de una sepsis y nefropatía obstructiva por cristalización en los túbulos renales del Aciclovir (15). En nuestro enfermo, la insuficiencia renal podría explicarse en el contexto de una glomerulonefritis secundaria a la viriasis o como efecto adverso del Aciclovir por exclusión de las otras causas. Por otro lado, aunque la hepatitis es poco común, no son infrecuentes las elevaciones mínimas de las transaminasas $(16,17)$. También es frecuente la trombocitopenia autolimitada atribuida a un doble mecanismo: acción tóxica directa del virus sobre las plaquetas en una fase inicial y mediada por anticuerpos antiplaquetas en fases posteriores (18). Tanto las elevaciones de las transaminasas como la trombocitopenia aparecieron en el caso aportado. Podemos concluir que en todo adulto con varicela es conveniente realizar una radiografía de tórax y un seguimiento hasta la resolución del rash, con especial atención a la aparición de manifestaciones respiratorias y siempre teniendo en cuenta otro tipo de complicaciones que en muchas ocasiones pasan desapercibidas debido a las mínimas manifestaciones clínicas con las que cursan (19). 


\section{Bibliografía}

1. Laufenburg HF. Varicella Pneumonia: A case report and review. Am Fam Phys 1994; 50 (4): 793-6.

2. Schlossberg D, Littman M. Varicella Pneumonia. Arch Intern Med 1988; 148: 1630-2.

3. Sans M, Gatell JM, Rafael M, Mallolas J, Soriano E. Neumonía varicelosa en adultos infectados por el VIH-1. Presentación de 2 casos. Enferm Infecc Microbiol Clin 1994; 12: 26-30.

4. Waring JJ, Neubuerger K, Greever EF. Severe form of chickenpox in adults. Arch Intern Med 1942; 69: 384-408.

5. Davidson RN, Lynn W, Savage P, Wansbrough-Jones MH. Chickenpox pneumonia: experience with antiviral treatment. Thorax 1988; 43: 627-30.

6. Quintana González JI, Rodríguez Pascual L, Morato Arnáiz A, García Arroyo I, Martín Torre E. Neumonía varicelosa en adultos sanos. A propósito de 6 casos. Arch Bronconeumol 1996; 32: 369-72.

7. Meyer B, Stalder H, Wegmann W. Persistent pulmonary granulomas after recovery from varicella. Chest 1986; 89 (3): 457-9.

8. Rodrigues J, Niederman MS. Pneumonia complicating pregnancy. Clin Chest Med 1992; 13: 679-91.

9. Wallace MR, Bowler WA, Murray NB, Brodine SK, Oldfield EC. Treatment of adult varicella with oral Acyclovir. A randomized, placebocontrolled trial. Ann Intern Med 1992; 117: 358-63.

10. García Quintana A, Alegre Martín J, Falcó V, Fernández de Sevilla T, Martínez Vázquez JM. Neumonía varicelosa en el adulto. Estudio de trece casos. Rev Clin Esp 1992; 191: 314-6.
11. Roberts DE. J Am Board Fam Pract 1995; 8 (1): 52-4.

12. Pitel PA, McCormick KL, Fitzgerald E, et al. Subclinical hepatic changes in varicella infection. Pediatrics 1980; 65: 631-3.

13. Rubio Félix SA, Val Adán P, Escobedo Palau JA, Sebastián Royo M, Borderías Clau L, Garrapiz López J. Neumonitis varicelosa en el adulto previamente sano. Arch Bronconeumol 1994; 30: 468-70.

14. García Quintana A, Alegre Martín J, Falcó V, Fernández de Sevilla T, Martínez Vázquez JM. Neumonía varicelosa en el adulto. Estudio de trece casos. Rev Clin Esp 1992; 191: 314-6.

15. Roberts DE J. Am Board Fam Pract 1995; 8 (1): 52-4.

16. Pitel PA, McCormic KL, Fitzgeral E, et al. Subclinical hepatic changes in varicella infection. Pediatrics 1980; 65: 631-3.

17. Barreiro García PM, Pintor Holguín E, Burón Fernández MR, Díaz Pollán B, Roca Arbonés V. Neumonitis asociada a primoinfección por virus varicela-zoster: A propósito de 11 casos (carta). An Med Interna (Madrid) 1999; 16: 209-10.

18. Rubio Félix SA, Val Adán P, Escobedo Palau JA, Sebastián Royo M, Borderías Clau L, Garrapiz López J. Neumonitis varicelosa en el adulto previamente sano. Arch Bronconeumol 1994; 30: 468-70.

19. Golpe Gómez R, García Arangüena L, García Pérez MM, De la Roza Fernández CO, Fernández Rozas SM, Cifrián Martínez JM. Neumonía varicelosa en el adulto previamente sano. An Med Interna (Madrid) 1999; 16: 83-6. 